Editorial

\title{
Cyclic gmp-amp synthase: a new putative antituberculosis immune enzyme
}

Volume I Issue 3 - 2015

\section{Editorial}

In a study published in the journal of Cell Host \& Microbe by Shiloh et al., ${ }^{1}$ It has recently become apparent that pathogen directed immune cell can produces and may be secretes an identifiable e enzyme involved in protecting the body from different pathogens having a remarkable ability to senses Mycobacterium tuberculosis (TB), the most powerful killer of a bacterial pathogen that can leave 1.5 million deaths annually with an estimated of millions of infected people worldwide.

The Team researchers stated "that the new finding has potential implications for the development of immunity-based therapies to treat tuberculosis". This development, may be, is inevitable in the salvation of the poor patients from a prolonged agonized 9month Treatment and from a serious complications of this disease.

This signaling enzyme, The researchers explained, called cyclic GMP-AMP synthase (cGAS), exerting it's unique sensor effects in immune defense stream against the bacteria in general and tuberculosis bacteria in particular. The mechanisms possibly responsible for this, and in host defense against M. tuberculosis. However, the identity of the host sensors for, is completely unknown.

However there is no denying that Activation of the DNAdependent cytosolic pathway is an important factor which plays an important role in response to Mycobacterium tuberculosis infection. The activation of the above cytosolic pathway at any given level of phagocytic - cell Function, directed against this bacteria, by stimulating ubiquitin-dependent autophagy and inflammatory cytokine production specifically type I interferon. This well-known mechanism could not be explained by the absence of unknown sensor M. tuberculosis DNA, so this study put forth a new putative enzyme sensor.

No need to state that the only licensed and long introduced TB vaccine is bacillus Calmette Guérin (BCG), which a live attenuated vaccine successfully derived from Mycobacterium bovis very long time ago. It is the oldest and most widely used vaccine worldwide. Although the BCG from an immunological point view is a potential one that can induce potent cellular immune responses in infants and children, and also protects effectively against disseminated TB in children, but the efficacy and duration of protection is questionable in adults in which many vaccinated adults showing only variable protection against the disease suggesting a diminish state in cellular immune responses. Furthermore, BCG vaccine cannot destroy latent bacteria in pulmonary $\mathrm{TB}$ and prevent subsequent $\mathrm{TB}$ infection.

\author{
Chateen Izaddin Ali Pambuk \\ University of Tikrit, Ira
}

Correspondence: Chateen Izaddin Ali Pambuk, University of Tikrit/College of Dentistry Iraq, Tel 07701808805, Email dr.chatin2@yahoo.com

Received: December 12, 2015 | Published: December 29, 2015

New techniques in vaccination, moreover have offered to develop a new vaccines constructed to be modified toxins, protein conjugated polysaccharides and what is called immunoreactive proteins, substantially proven to be protective in animal models. In addition, the breakthrough of genomics has provided novel methods for selecting antigens useful to build up new protective anti-TB vaccines.

A crucial question posed in this context is this new enzyme, if it is possible to be converted to a potent vaccine, could alter the developing state of $\mathrm{Tb}$ vaccination among the elegant approaches nowadays??

\section{Acknowledgments}

None.

\section{Conflicts of interest}

Author declares there are no conflicts of interest.

\section{Funding}

None.

\section{References}

1. Angela CC, Haocheng C, Zhijian JC, et al. Cyclic GMP-AMP Synthase Is an Innate Immune DNA Sensor for Mycobacterium tuberculosis. Cell Host \& Microbe. 2015;17:1-9. 